\title{
Optical loss and crosstalk in multimode photolithographically- fabricated polyacrylate polymer waveguide crossings
}

\author{
Hadi Baghsiahi*, Kai Wang, David R. Selviah \\ Department of Electronic and Electrical Engineering,University College London (UCL),Torrington \\ Place, London, WC1E 7J, UK, +44(0)2076794156 \\ h.baghsiahi@ee.ucl.ac.uk, d.selviah@ee.ucl.ac.uk
}

\begin{abstract}
Complex interconnection patterns in electrical PCBs have to use multiple layers of copper tracks. However, the same interconnections can be made in a single layer using optical waveguides as they cross on the same layer. Waveguide crossings where two waveguides intersect in the same optical layer are particularly important components as they offer OPCB layout designers additional flexibility to solve layout problems such as routing around cutout areas, electrical components and other obstacles on an OPCB. Use of waveguide crossings can also help to avoid sharp bends in the design as these bends are an important cause of optical loss. Despite all of the advantages of waveguide crossings, and although most of the light travels along the intended waveguide, a proportion of the optical power in one waveguide will couple into the crossing waveguide at each intersection point or couple out of the original waveguide and into the cladding. This coupling phenomenon causes optical loss and crosstalk in the system.

In this paper, the results of an investigation of the optical loss due to the crossing of multimode polymer waveguide, fabricated on FR4 printed circuit boards, PCBs, as a function of crossing angles are presented theoretically and experimentally. The results from ray tracing simulation is compared with the experiment results and the contrast is discussed.
\end{abstract}

Keywords: Optical waveguide, waveguide crossing, optical loss, optical interconnect, OPCB, high data rate, multimode waveguide, crossovers, layout, routing, datacoms, OCB.

\section{INTRODUCTION}

The optical part on an OPCB can be more compact and have fewer layers than the electrical part as optical signals can cross each other without suffering from severe crosstalk. Crossings where two or more waveguides intersect in the same optical layer become critical components in the optical design of the OPCB layout. Waveguide crossings are useful and can be applied in the design of OPCB boards as waveguides need to route around cutout areas, electrical components and other obstacles on an OPCB [1;2]. It will also help to avoid sharp bends in the design as bent waveguides are a principal source of optical loss [1;3]. Waveguide intersections can occur in several possible configurations, for example; straightstraight, straight-bend and bend-bend. Despite the benefits of using crossing waveguides, and although most of the light travels along the intended waveguide, a proportion of the optical power in one waveguide is coupled into the crossed waveguide at each intersection point, especially for small crossing angles (Figure 1). This coupling phenomenon causes optical loss and crosstalk in the system which is dependent on the crossing angle between the waveguides [4;5]. Apart from the optical energy which is coupled into the crossing waveguide, some of the optical energy is lost by entering the cladding area when beams do not satisfy the total internal reflection (TIR) criterion. Consequently the crossings in OPCBs need to be carefully designed to reduce both loss and crosstalk and to increase the performance of the system by reducing the bit error rate.

Optical loss at waveguide crossings can be explained by geometrical optics and wave optics. Considering Figure 1 and based on wave optics, the optical power is distributed between the bound modes inside the horizontal waveguide. Waveguide crossings cause a change in the modal distribution of light travelling at the crossing as the waveguide structure and so the boundary conditions change at the crossing [6]. Some of the power contained within a waveguide may be coupled to radiation modes [6] and, hence, be coupled into the cladding or recoupled into another waveguide or. From a geometrical point of view, when the incoming or internal reflected light inside the waveguide reaches the intersection point, the incident angles are larger than the critical angle of the waveguides due to the different geometry at the crossing part and the optical power in the rays, which do not satisfy total internal reflection condition, will be lost.

Integrated Optics: Devices, Materials, and Technologies XVIII, edited by Jean Emmanuel Broquin,

Gualtiero Nunzi Conti, Proc. of SPIE Vol. 8988, 898807 - (c) 2014 SPIE

CCC code: $0277-786 \mathrm{X} / 14 / \$ 18 \cdot$ doi: $10.1117 / 12.2039860$ 
For the crosstalk, all of the light that enters the branch waveguide at the incident angles less than the critical angle of the branch waveguides will continuously propagate along the branch waveguide. (All waveguides are made of the same core and cladding materials and have the same TIR condition).

The optical loss due to several number of $90^{\circ}$ crossings has been tested by experiment and simulation[4;7-9]. However, most of the previous research is on high index difference silicon based waveguides for semiconductors. In addition, no one has considered the optical loss due to the waveguide crossing by both simulation and experiment and explained the factors affecting the loss.

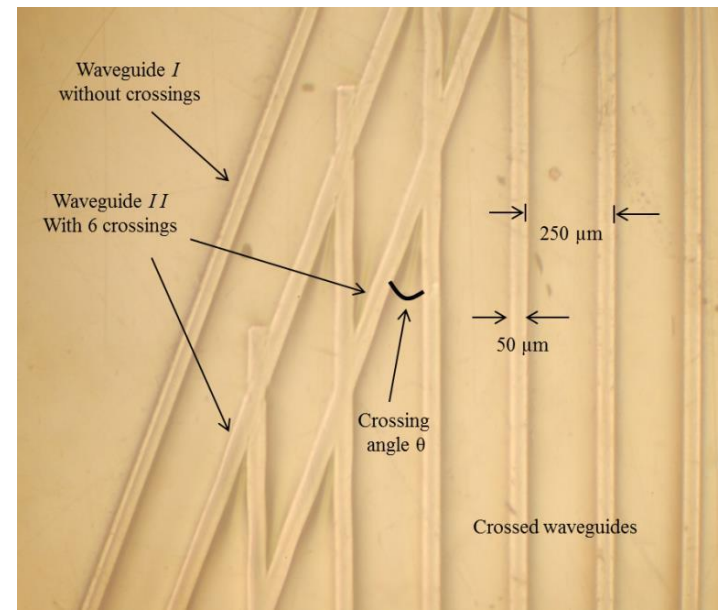

Figure 1: Photograph of $50^{\circ}$ waveguide crossings taken with a Nomarski microscope using front illumination

\subsection{Computer modeling of the waveguide crossing}

The crossing waveguides are simulated using ray tracing technique. The simulated structure is a channel waveguide with a cross-section of $50 \mu \mathrm{m} \times 50 \mu \mathrm{m}$. The wavelength of the source is $853 \mathrm{~nm}$ and the refractive indices of the core and the cladding are chosen (based on the minimum absorption loss $853 \mathrm{~nm}$ ) to be 1.5560 and 1.5264 respectively; the simulation parameters were chosen to be as close as possible to the manufactured waveguide for comparison with the experimental results. Figure 1 shows a photograph of a set of $50^{\circ}$ crossings and a straight reference waveguide which was used in the experimental measurement of optical loss due to the crossing waveguides explained in the next section.

\subsection{Simulating Crossings with Ray Tracing}

Simulation of waveguide crossings using wave optics has some limitations, in particular, with respect to the crossing angle. If we were to solve Maxwell's equations in full vectorial mode, the amount of memory and calculation time is significantly increased. Classical geometrical optics where light is considered as rays or particles rather than waves, can be used for simulation. In this method, the rays are traced through the system starting from the light source and change direction when they pass through an object with a different refractive index [10]. In the waveguide, millions of rays (defined by the user) are emitted from the source depending on its design, and each ray is traced inside the waveguide. The ray tracing method uses total internal reflection and Fresnel equations to trace the rays and calculates the number of rays that are detected at the desired detector. In terms of simulation by computer, ray tracing programs require less memory and are much faster (a calculation which takes 2 days in BPM, takes 10 minutes in ray tracing) and there is no limitation on the crossing angle.

The schematic diagram in Figure 2 shows a simple simulation model for waveguide crossings using the ray tracing method. A crossing structure basically contains two straight $50 \mu \mathrm{m} \times 50 \mu \mathrm{m}$ waveguides that intersect each other at an angle between 0 to $90^{\circ}$. The waveguides have a core layer inside a cladding layer. The core has a relatively higher refractive index of 1.5560 while the cladding's refractive index is 1.5264 in order to guide the light. 


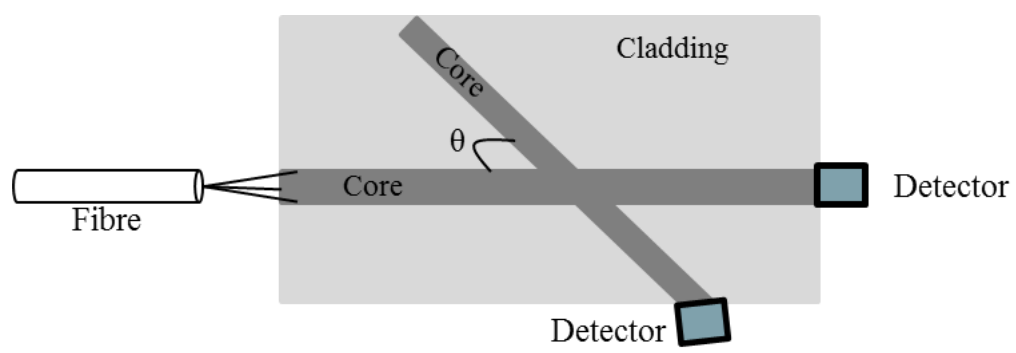

Figure 2. Schematic diagram of the crossing waveguide

The source used in this simulation is a step index multimode fibre with a core diameter of $50 \mu \mathrm{m}$ at a wavelength of 853 $\mathrm{nm}$ and $\mathrm{NA}=0.22$ which matches the NA of the fibre used for the experiment.

The length of each straight section is $100 \mathrm{~mm}$ and the input fibre source is placed 1-2 $\mu \mathrm{m}$ away from the entrance of the waveguide. $1-2 \mu \mathrm{m}$ is the distance between the fibre as the source and the waveguide in the experiment. The intersection takes place at the centre of the two waveguides. This distance is after the equilibrium length [11] and the rays are confined in the core area before arriving at the crossing section. Two detectors are placed at the end of each waveguide to measure the power output from each waveguide. One detector also records the power just before the crossing section in the main waveguide. This detector is placed after the equilibrium length and close to the crossing section. Power is measured by counting the number of rays that impinge on the detector surface. The power of the source is set to be 1 $\mathrm{mW}$ and 1,000,000 rays are generated from this source and power loss is simulated at crossing angles from $10^{\circ}$ to $90^{\circ}$ in steps of $10^{\circ}$. Figure 3 shows the results of the ray tracing simulation for the waveguides with various crossing angles. The NA of the fibre as the light source was chosen to be 0.22 and the optical loss for crossing with different angle was found. Figure 3 shows the results for the waveguides with various crossing angles. The results show a high magnitude of loss (more than one $\mathrm{dB}$ ) for sharp angles (less than $20^{\circ}$ )

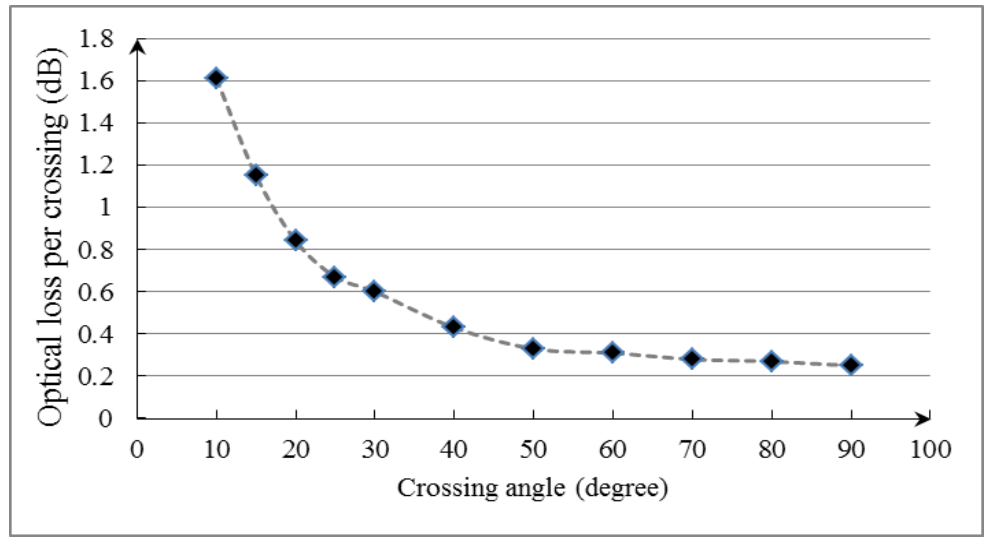

Figure 3. Ray tracing results for simulation of crossing waveguides with different crossing angles

\section{EXPERIMENTAL RESULTS}

\subsection{Experiment configuration}

In this section the experimental results of the optical loss in crossing waveguides are presented. The light source used is an $853 \mathrm{~nm}$ VCSEL launched into a standard 50/125 $\mu \mathrm{m}$ step index multimode fibre (NA = 0.22). The fibre source was aligned and butt-coupled to the waveguide. The optical loss due to the waveguide crossing were measured for the crossing angles from $10^{\circ}$ to $30^{\circ}$ in increments of $5^{\circ}$, and $40^{\circ}$ to $90^{\circ}$ in increments of $10^{\circ}$ giving 11 crossing angles in total. However, as only the loss purely due to the crossing structure is of interest, all the optical losses due to the material absorption were excluded from the experiment results to achieve the optical loss just due to the crossing by arranging the experiment below. 


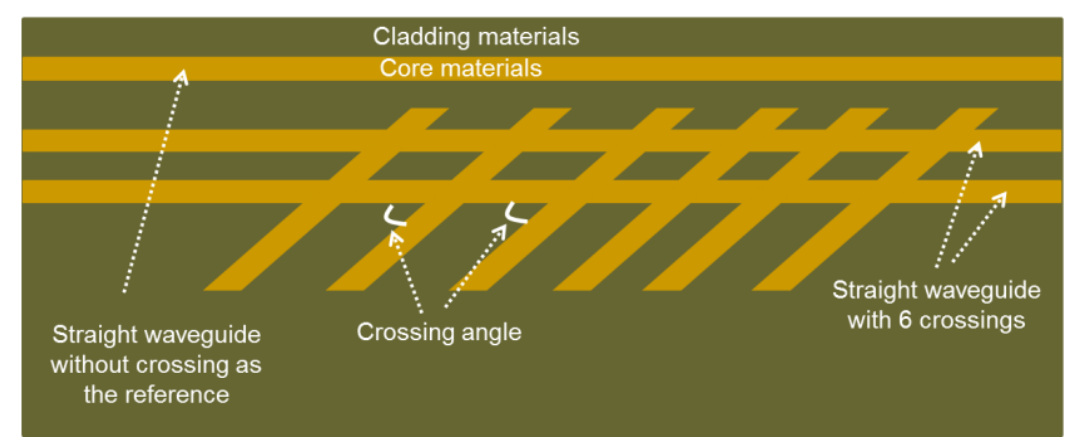

Figure 4. Schematic diagram of the crossing waveguides used for measuring the optical loss caused by the waveguide crossings. The size of the waveguides was designed to be $50 \mu \mathrm{m}$

Figure 4 shows a schematic diagram of the waveguides. Three identical straight waveguides were fabricated on an FR4 substrate. 2 waveguides out of these three were crossed by 6 identical waveguides. Therefore, there were 6 crossings for each crossing angle. Since the 3 straight waveguides were exactly the same, the output power of these straight waveguides was expected to be equal. Hence, when crossing waveguides are added into one of the straight waveguides, the difference in output power between the straight waveguides with and without crossings is purely due to the crossing waveguides. The optical loss for each crossing angle was measured 50 times as the input power was set to be $0 \mathrm{dBm}$ and the output power was low $(-10 \mathrm{~dB})$ and the measurement was repeated to reduce the error by averaging 50 measurements. The crossing angles were; $10^{\circ}, 15^{\circ}, 20^{\circ}, 25^{\circ}$, and from $30^{\circ}$ to $90^{\circ}$ in increments of $10^{\circ}$, giving a total of 11 different angles. The optical power loss (in $\mathrm{dB}$ ) detected at the end of the waveguide without crossings was subtracted from the optical loss detected at the end of the waveguide with crossings to find the optical loss due to the 6 crossings (The input power for this experiment was arranged to be $0 \mathrm{dBm}$ ). The mean value of the optical loss for each crossing angle in the 50 measurements was divided by 6 to find the optical loss due to one crossing.

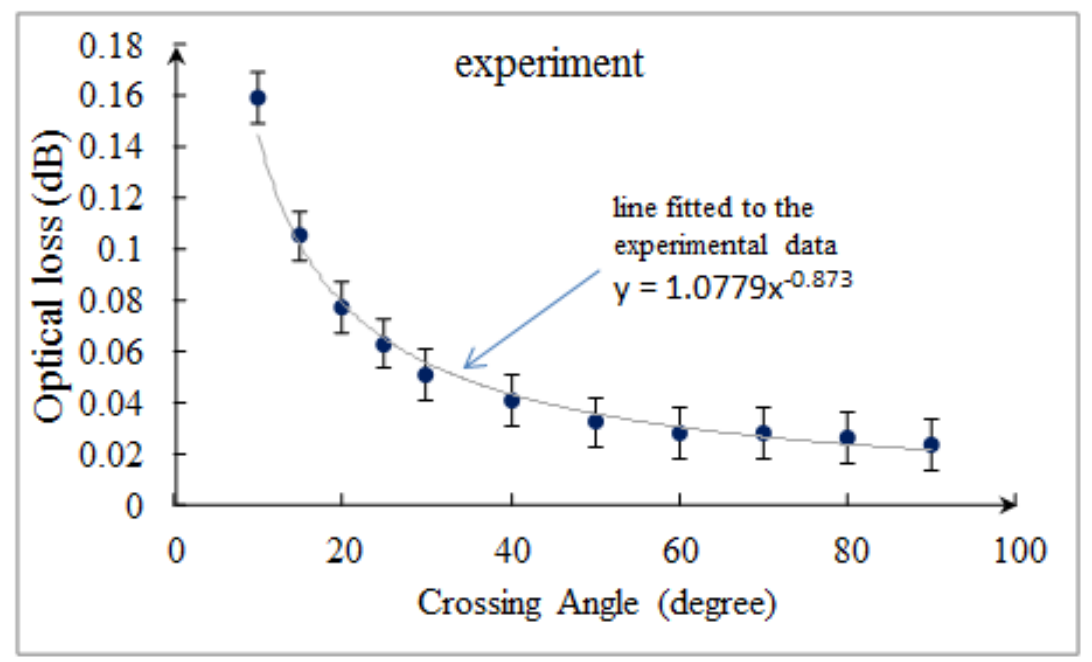

Figure 5. Experiment results for the optical loss per crossing for different crossing angle

Figure 5 shows the optical loss measurement for each crossing versus the crossing angles. The vertical axis in this figure is the optical loss per crossing which is defined as the power measured at the end of the straight waveguide containing 6 crossings divided by 6 .

\section{DISCUSSION OF THE RESULTS OF CROSSING WAVEGUIDE SIMULATION AND EXPERIMENT}

The simulation results from ray tracing method are compared with the experiment results in Figure 6. The results show a large difference between the experimental and the ray tracing simulation results. 
Figure 6 shows the ray tracing simulation results and the experimental results and it can be seen that the simulation results predict a much larger optical loss for each crossing angles.

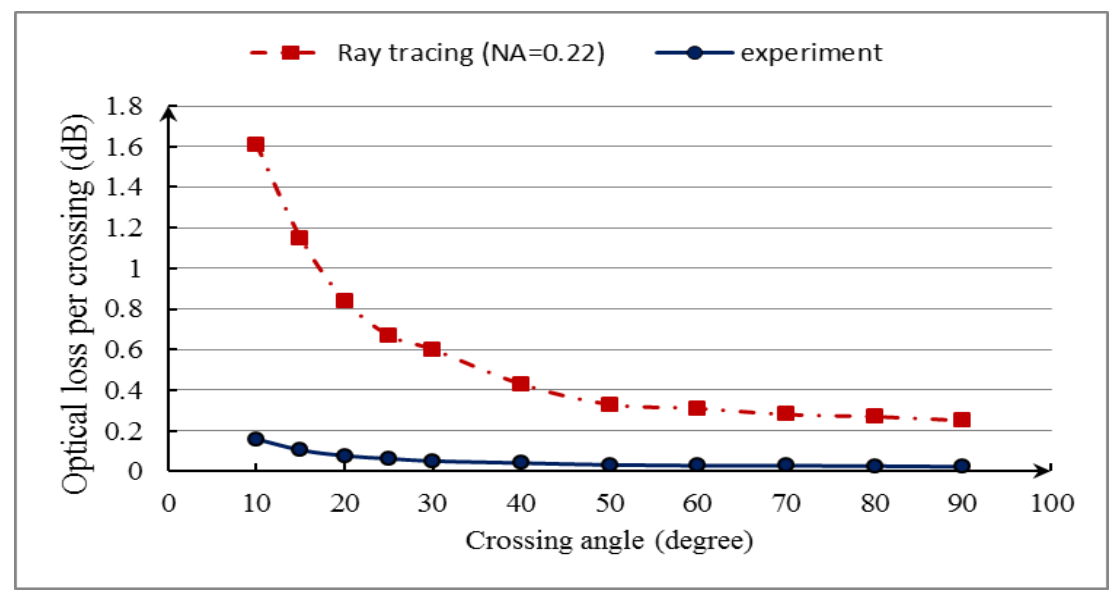

Figure 6. Optical loss of one crossing as a function of the angle of the crossing for a calculation using ray tracing compared to the experimental result. The input source has an $\mathrm{NA}=0.22$

The difference in the optical loss measured by experiment and calculated by ray tracing method is shown in Figure 7 . The light source for the simulation is an optical fibre with $50 \mu \mathrm{m}$ core diameter and $\mathrm{NA}=0.22$, which is the same as for the experiments. The difference between the simulation and the experiments must be considered; for example ray tracing assumes a rectangular waveguide with smooth walls and no wall roughness.

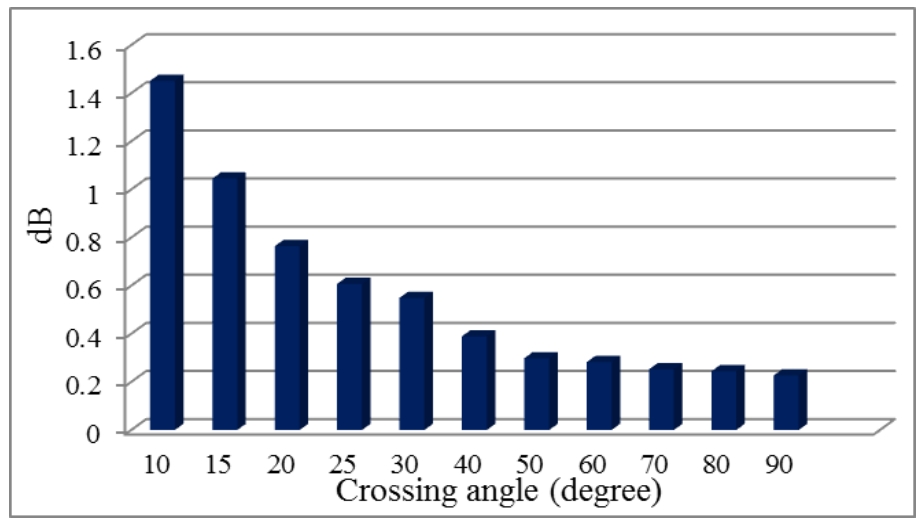

Figure 7. Difference between the optical loss in ray tracing simulation and the experiments. The input source for the simulation is a fibre with $50 \mu \mathrm{m}$ core diameter and $\mathrm{NA}=0.22$

The ZEMAX software traces millions of rays in a cone for $\mathrm{NA}=0.22$ inside the waveguide and then follows them until they strike the detector or are lost. Therefore, any ray which is subject to total internal reflection will be guided inside the waveguide. The NA of the fibre indicates that rays are emitted in a cone with half angle of $12.70^{\circ}$ and Snell's law determines the maximum angle inside the waveguide is $8.1^{\circ}$ which means the incidence angle on the core cladding interface is $81.9^{\circ}$. These numbers were calculated based on the numerical aperture formula for a waveguide[6].

$$
\begin{gathered}
N A=n \sin \theta=\sqrt{n_{\text {core }}^{2}-n_{\text {clad }}^{2}} \\
\sin \theta_{c}=\frac{n_{\text {clad }}}{n_{\text {core }}}
\end{gathered}
$$

Where $n$ is the refractive index of the medium outside the waveguide/fibre and, $\theta_{c}$ is the critical angle of the waveguide/fibre. The critical angle of the waveguide is $78^{\circ}$ so that any ray which enters the waveguide will propagate 
within it. The difference from an actual waveguide is the existence of wall roughness along the waveguides which causes scattering to radiation modes which are lost [3;12-14]. Therefore, only rays with a shallow angle (low order modes) propagate in the waveguide. To determine the range of angles which propagate the output of the waveguide was measured; the measurement shows that the maximum output angle is about $7^{\circ} \pm 2^{\circ}$.

To simulate the low order mode inside the waveguide the NA of the source was reduced to find the smallest NA which shows optical loss comparable to the experiments. The best results were achieved where the NA $=0.05$. The results are shown in Figure 8 where the loss in the experiment is the same magnitude as the simulation when experimental error is taken into account.

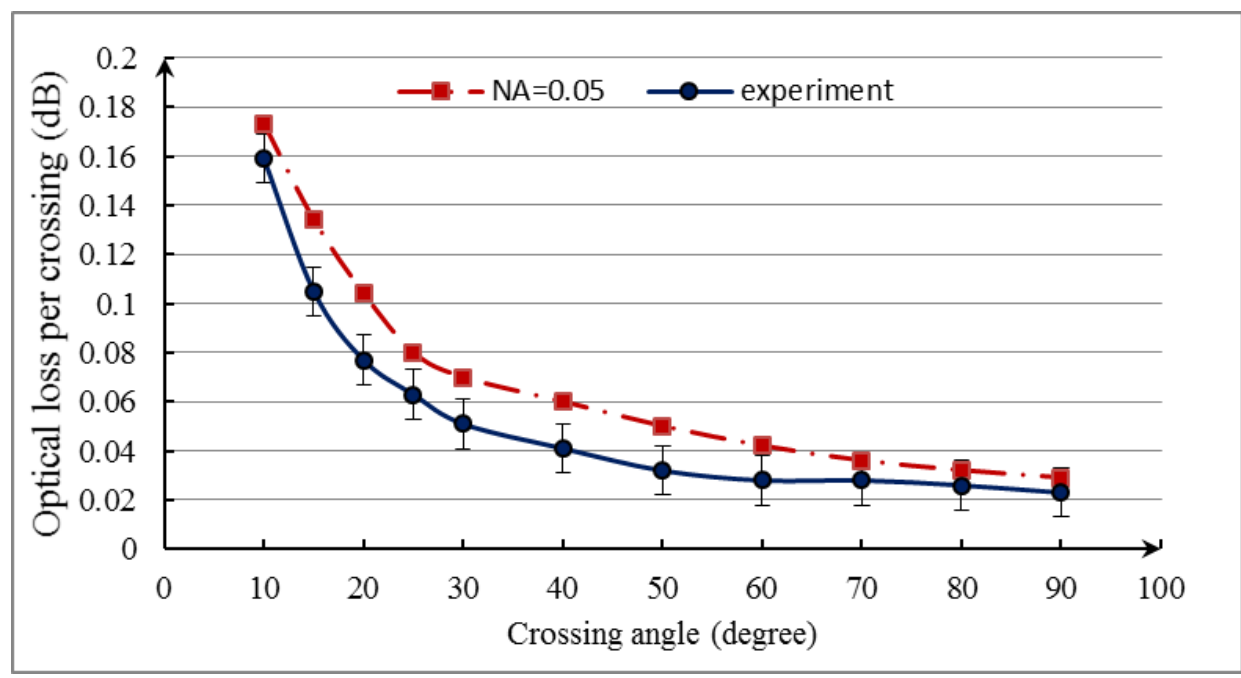

Figure 8. Experimental results and the ray tracing results for the source with $\mathrm{NA}=0.05$.

\section{CONCLUSIONS}

In this paper, the optical loss due to crossing waveguides was calculated for different crossing angles. The simulation results were compared with the experiment results and results difference were investigated. Most of the previous research on crossings is for a different material, such as silicon waveguides, or just for $90^{\circ}$ crossings.

Ray tracing methods were used for the waveguide simulations. To find the source of this error, the process of optical loss at the crossing was reconsidered. The optical source for the simulation was an optical fibre with NA of 0.22 and the waveguide NA is 0.3 . The critical angle of the waveguide is $78^{\circ}$ so that any ray which enters the waveguide will be propagated within the waveguide. In an actual waveguide wall roughness causes beams with sharper angles (high order modes) to radiate away from the waveguide. Therefore, only rays with a shallow angle (low order modes) are propagated in the waveguide. To determine the range of angles that propagate inside the waveguide, the NA of the source was reduced to find the smallest NA which shows optical loss comparable to the experiments. The best results were achieved where the NA $=0.05$. This NA corresponds to an angle of 6 degrees. The interesting result is that the output angle of the waveguide was measured to be $7^{\circ} \pm 2^{\circ}$. 


\section{REFERENCES}

[1] K. Wang, D. R. Selviah, I. Papakonstantinou, G. Yu, H. Baghsiahi, and F. A. Fernandez, "Photolithographically manufactured acrylate polymer multimode optical waveguide loss design rules," Electronics System-Integration Technology Conference, ESTC 2008, pp. 1251-1256, 2008.

[2] R. Pitwon, K. Wang, J. Graham-Jones, I. Papakonstantinou, H. Baghsiahi, B. Offrein, R. Dangel, D. Milward, and D. Selviah, "FirstLight: Pluggable Optical Interconnect Technologies for Polymeric Electro-Optical Printed Circuit Boards in Data Centers," IEEE Journal of Lightwave Technology, vol. 30, no. 21, pp. 3316-3329, Nov. 2012.

[3] I. Papakonstantinou, K. Wang, D. R. Selviah, and F. Anibal Fernandez, "Transition, radiation and propagation loss in polymer multimode waveguide bends," Optics Express, vol. 15, no. 2, pp. 669-679, 2007.

[4] M. G. Daly, P. E. Jessop, and D. Yevick, "Crosstalk reduction in intersecting rib waveguides," Lightwave Technology, Journal of, vol. 14, no. 7, pp. 1695-1698, 1996.

[5] P. Sanchis, P. Villalba, F. Cuesta, Anderas Hakansson, and Amadeu Griol., "Highly efficient crossing structure for silicon-on-insulator waveguides," Optics Letters, vol. 34, no. 18, pp. 2760-2762, 2009.

[6] A. W. Snyder and J. D. Love, Optical waveguide theory, 4 ed Springer, 1983.

[7] P. J. Bock, P. Cheben, J. H. Schmid, J. Lapointe, D. X. Xu, S. Janz, A. Densmore, and T. J. Hall, "Subwavelength grating crossings for silicon wire waveguides," Optics Express, vol. 18, no. 15, pp. 16146$16155,2010$.

[8] W. Bogaerts, P. Dumon, D. V. Thourhout, and R. Baets, "Low-loss, low-cross-talk crossings for silicon-oninsulator nanophotonic waveguides," Optics Letters, vol. 32, no. 19, pp. 2801-2803, 2007.

[9] T. Ishigure, K. Shitanda, T. Kudo, S. Takayama, T. Mori, K. Moriya, and K. Choki, "Low-loss design and fabrication of multimode polymer optical waveguide circuit with crossings for high-density optical PCB," IEEE, 2013, pp. 297-304.

[10] Eugene Hecht and Alfred Zajac, Optics, 4 ed Addison Wesley, 1990.

[11] L. B. Soldano and E. C. Pennings, "Optical multi-mode interference devices based on self-imaging: principles and applications," Lightwave Technology, Journal of, vol. 13, no. 4, pp. 615-627, 1995.

[12] K. Wang, D. R. Selviah, J. Papakonstantinou, G. Yu, H. Baghsiahi, and F. A. Fernandez, "Photolithographically manufactured acrylate polymer multimode optical waveguide loss design rules," Proceedings - 2008 2nd Electronics Systemintegration Technology Conference, ESTC, pp. 1251-1255, 2008.

[13] H. Baghsiahi, K. Wang, W. Kandulski, R. C. Pitwon, and D. R. Selviah, "Optical Waveguide End Facet Roughness and Optical Coupling Loss," Lightwave Technology, Journal of, vol. 31, no. 16, pp. 2659-2668, 2013

[14] V. Celli, A. Marvin, and F. Toigo, "Light-Scattering from Rough Surfaces," Bulletin of the American Physical Society, vol. 19, no. 10, p. 1081, 1974. 